\title{
Constelaciones Familiares: ¿Psicoterapia o pseudoterapia? Revisión de la literatura desde la filosofía de la ciencia
}

\section{Family Constellation: Psychotherapy or pseudo-therapy? Literature review from the philosophy of science}

DOI: $10.46932 / \mathrm{sfjdv} 2 \mathrm{n} 2-180$

Received in: March 1st, 2021

Accepted in: May 30th, 2021

\section{Cristhian Javier Rivera Tapia \\ Psicólogo Clínico}

Institución: Unidad de Posgrados Universidad Católica de Cuenca

Dirección laboral: Practica privada - Ecuador, Azuay, Cuenca, El sagrario

Correo electrónico: c.riveratapia@ hotmail.com

\section{Álvaro Fabian Auquilla Guzmán \\ Psicólogo Clínico}

Institución: Unidad de Posgrados Universidad Catolica de Cuenca

Dirección laboral: Practica privada - Ecuador, Azuay, Cuenca, Yanuncay

Correo electrónico alfast2009@ hotmail.com

Xavier Fabricio Reyes Trelles

Psicólogo Clínico

Institución: Ministerio de Inclusión Económica y Social

Dirección laboral: Ecuador, Cañar, Azogues, Luis Cordero y Fray Vicente Solano

Correo electrónico: fabricioreyestrelles@gmail.com

\section{Braulio Andrés Elizalde Martínez}

Psicólogo Clínico

Institución: Fundación de Desarrollo Social de El Oro - FUDESO

Dirección laboral: Ecuador, El Oro, Machala, Cdla. Las Brisas

Correo electrónico: brau.elizalde@ gmail.com

\section{Walter Jacinto Portoviejo Fernández}

Psicólogo clínico.

Institución: Unidad de Posgrados de la Universidad Católica de Cuenca.

Dirección laboral: Ecuador, Azuay, Gualaceo, Luis Ríos Rodríguez y Antonio Vera.

Correo electrónico: walterportoviejo@yahoo.com

\section{Freddy Alejandro Castro Salinas \\ Psicólogo Clínico}

Institución: GAD Municipal Sígsig.

Dirección laboral: Calles 3 de noviembre y Juan Pablo II.

Correo electrónico: alejandrocastro2295@gmail.com

Pedro Carlos Martínez Suarez

$\mathrm{PhD}$ en Psicología. 
Institución: Laboratorio de Psicometría, etología y Psicología comparada. Universidad Católica de Cuenca, Campus CIITT.

Dirección personal: Ecuador, Azuay, Cuenca, parroquia Ricaurte, Vía Ricaurte Bibín frente a Plásticos Rival.

Correo electrónico: pmartinezs@ucacue.edu.ec

\title{
RESUMEN
}

La búsqueda de efectividad para los procesos psicoterapéuticos destaca la necesidad de abordar, desde una perspectiva científica, aquellos modelos que se encuentran en el margen de la Psicología. La práctica de constelaciones familiares, impulsada en el trabajo de Bert Hellinger, implica procedimientos a veces asociados a la labor psicoterapéutica. Por esto, resultó pertinente elaborar una revisión crítica de la literatura internacional actualizada de 21 artículos de interés publicados sobre la temática, principalmente aquellos centrados en su procedimiento y efectividad como recurso de curación. La información obtenida fue revisada a través de los postulados ontológicos de la epistemología de Bunge sobre la realidad científica. A partir de las investigaciones, cuantitativas y cualitativas, no se encontraron resultados concluyentes respecto a su efectividad, esto asociado a carencias metodológicas y teóricas, además de evidenciarse conflictos en su relación con la Psicología y Psicoterapia, principalmente en sus supuestas bases de carácter sistémico y fenomenológico.

Palabras clave: constelaciones familiares, pseudociencia, terapia basada en la evidencia, sistémica, filosofía de la ciencia, psicoterapia, validación.

\begin{abstract}
Constant research for effectiveness in Psychotherapeutic processes highlights the need to address, from a scientific perspective, models that are positioned on the margins of Psychology. The practice of family constellations, as developed by Bert Hellinger, implies procedures sometimes associated with Psychotherapeutic work. For this reason, it was pertinent to elaborate a critical review of the updated international literature on 21 relevant articles published around this subject, mainly those focused on its procedure and effectiveness as a healing resource. The information obtained was reviewed through Bunge's ontological postulates for philosophy of science. No conclusive results were found regarding its effectiveness, this can be associated to methodological and theoretical deficiencies, also there are conflicts founded in its relation with the fields of Psychology and Psychotherapy, mainly in its supposed systemic and phenomenological foundations.
\end{abstract}

Keywords: family constellations, pseudoscience, evidence-based therapy, systemic, philosophy of science, psychotherapy, validation.

\section{INTRODUCCIÓN}

Desde sus inicios la psicología aplicada ha intentado establecer una asociación profunda con procedimientos basados en evidencia para el cuidado de pacientes (APA Presidential Task Force on Evidence-Based Practice, 2006). La psicoterapia ha recorrido un amplio camino buscando brindar un modelo efectivo de tratamiento a los trastornos mentales, así, pertenece a una clase de prácticas de curación que abordan diversas expresiones de angustia psicológica, en muchos sentidos, es amorfa pues está asociada a una serie de prácticas afines impartidas por diversos profesionales como psicólogos, 
psiquiatras o terapeutas matrimoniales, también se vale de una variedad de técnicas basadas en diferentes modelos teóricos (Wampold, 2019).

La existencia de múltiples modelos, unos más cercanos que otros a la cuestión de la evidencia, genera que existan prácticas en los márgenes, cuya pertenencia se discute en coyunturas importantes (Wampold, 2019). Mucha información pertinente para la práctica de psicoterapia se mantiene pendiente a ser descubierta o validada, y en ese margen de incertidumbre una serie de prácticas y modelos cuestionables han encontrado justificante para autodenominarse procesos psicológicos, psicoterapéuticos o de curación efectiva.

Es por esto, que la psicoterapia basada en evidencia (PBE), mantiene el objetivo de demostrar la eficacia de las aplicaciones terapéuticas y establecer parámetros claros en el abordaje de problemas mentales específicos, en lugar de darlos por sentado (Pérez, 2019). Así, la necesidad de actualizar información científica basada en evidencias sobre modelos en los márgenes de la psicología vuelve imperativo al abordaje de la práctica denominada Constelaciones Familiares $(\mathrm{CF})$, la cual desde su historia relativamente corta se ha establecido como un espacio de curación, muchas veces asociándose a procesos psicológicos y psicoterapéuticos.

Las CF parten de la teoría y práctica de Bert Hellinger (Stones, 2006), sus postulados buscan ubicarle en la categoría de terapia breve orientada en soluciones o sistémica (Franke, 2003). Se proclama sistémica, pues propone que desde la familia las dinámicas básicas, inconscientes y transmitidas a través de generaciones, pueden llevar a varias enfermedades y malestar psicológico (Hellinger, 1999). También, proponen características clave u órdenes que ayudan al flujo en un sistema, cuando es transgredido o afectado por un trauma, los miembros de la familia sufren, a menudo, dos o tres generaciones después de la ruptura (Stones, 2006).

Las constelaciones pretenden identificar rápidamente los enredos inconscientes en el sistema de origen y volverlos tratables, pues proponen que cambios en ese nivel conducen a cambios en el comportamiento, pensamiento y sentimientos (Franke, 2003). Durante su proceso, el facilitador se vale de las contribuciones de representantes para identificar cómo el alma familiar se ha enredado y ha interrumpido el flujo del orden. Hellinger observó que estos efectos transgeneracionales se manifestaban de varias maneras y utilizó una forma poética para describirlos (Stones, 2006).

En un inicio Hellinger define su práctica como una psicoterapia fenomenológica, argumentándola desde ese método filosófico. Esto significa para el facilitador someterse a contextos y conexiones más grandes sin la necesidad de comprenderlos, aceptar sin la intención de comprobar, y enfrentar lo que se presente de la manera en la que es (1999). Esta descripción implica que la CF no se basa en un modelo 
teórico, sino que sigue los fenómenos que surgen en la práctica, estos son, los mensajes no verbales del cliente (Franke, 2003).

Fenomenológicamente, un proceso terapéutico se centra en el pasado implícito que organiza y estructura el campo de relaciones del paciente (Fuchs, Messas y Stanghellini, 2019). En práctica, es un paradigma interpretativo, que funciona como filosofía y método de investigación (Wojnar y Swansosn, 2007). Dentro del campo de la psicoterapia este accionar implica que las formas de interrelación del paciente y la situación terapéutica se recrean "aquí y ahora" y pueden cambiarse mediante nuevas experiencias (Fuchs et al., 2019).

Definidas las características del modelo de CF, es pertinente revisar el contenido teórico, metodológico y los productos de investigación que han buscado acercarle a la PBE, discerniendo, de forma crítica y neutral, la información, por medio de la validez científica. Para ello, es necesario recurrir a la filosofía de la ciencia, pues como refiere Keplac (2012, citado por Pérez, 2019) "todas las actividades científicas se enraízan en asunciones filosóficas acerca del tipo de observaciones que constituyen los datos relevantes, los modelos causales y las consideraciones teóricas adecuadas. Variaciones en estas asunciones conducen a diferentes prácticas científicas” (p.14). Al hacerlo, se obtendrá una guía para reconocer la epistemología, validez de la terapia y diferenciarla de posturas pseudocientíficas.

Al definir el enfoque científico, Bunge (1985) refiere que la ciencia busca diferenciar, bajo criterios, lo que es científico de lo que no. Introduce el concepto de campos de conocimiento para caracterizar una actividad humana dirigida a obtener, difundir o utilizar información verdadera o falsa. Las ciencias deben cumplir con un conjunto determinado de características acorde con cada uno de los campos. Que la psicología cumpla con todos los criterios se torna difícil puesto que cuánto más riguroso se intenta ser, menos estudia el aspecto mental; y cuánto más se ocupe de lo mental, más riesgo de acercarse a la pseudociencia. De este modo, para analizar si un enfoque psicológico es pseudocientífico, Bunge (1985) propone analizarla bajo 5 de los 10 criterios: la filosofía inherente $(\mathrm{G})$, supuestos que toma de otros campos $(\mathrm{E})$, conjunto de problemas abordables $(\mathrm{P})$, los objetivos $(\mathrm{O})$, y la metódica $(\mathrm{M})$.

\section{METODOLOGÍA}

Se realizó una revisión crítica y sistemática de la literatura internacional de los artículos publicados en las bases datos Scopus, Web of Science, Research Gate y Google Scholar. Se aplicó un límite temporal de 20 años (2000-2020), debido a la escasa literatura. La selección de artículos consistió en una búsqueda bibliográfica exhaustiva en función de los siguientes criterios: estudios de tipo cuantitativo y cualitativo, revisiones bibliográficas. Se excluyeron estudios no accesibles a la totalidad del texto y entrevistas a 
profesionales. Posteriormente, se realizó una búsqueda mediante la modalidad bola de nieve, en las referencias de artículos seleccionados, a fin de localizar estudios no hallados en un primer momento.

El objetivo de la búsqueda bibliográfica fue obtener documentos que aporten información sobre la definición de $\mathrm{CF}$, proceso de aplicación y estudios que demuestren su efectividad en el tratamiento. Las palabras claves utilizadas fueron: Bert Hellinger, family constellation, systemic constellation, familienaufstellung. Se realizaron los siguientes truncamientos: Bert Hellinger and family constellations, Bert Hellinger and familienaufstellung. Es importante destacar que se realizó una desambiguación en el término family constellation puesto que en el idioma inglés también se usa para hacer referencia a la estructura familiar (véase en la Tabla 1).

Tabla 1 Registro de búsquedas realizadas

\begin{tabular}{|c|c|c|c|c|c|}
\hline Base de datos & $\begin{array}{l}\text { Palabras clave usadas en la } \\
\text { búsqueda }\end{array}$ & $\begin{array}{l}\text { Registros } \\
\text { encontrados }\end{array}$ & $\begin{array}{l}\text { Criterios de filtro: } \\
\text { año, relevancia }\end{array}$ & $\begin{array}{l}\text { Bola de } \\
\text { Nieve }\end{array}$ & Usados \\
\hline \multirow{5}{*}{ Scopus } & Bert Hellinger & 9 & \multirow{5}{*}{26} & \multirow{5}{*}{3} & \multirow{4}{*}{12} \\
\hline & Family Constellation & 2224 & & & \\
\hline & Systemic Constellation & 675 & & & \\
\hline & Familienaufstellung & 1 & & & \\
\hline & Bert Hellinger & 6 & & & \multirow{4}{*}{5} \\
\hline \multirow{3}{*}{$\begin{array}{l}\text { Web of } \\
\text { Science }\end{array}$} & Family Constellation & 1144 & \multirow{3}{*}{35} & \multirow{3}{*}{-} & \\
\hline & Systemic Constellation & 390 & & & \\
\hline & Familienaufstellung & 0 & & & \\
\hline $\begin{array}{l}\text { Research } \\
\text { Gate }\end{array}$ & $\begin{array}{c}\text { Bert Hellinger } \\
\text { Family Constellation } \\
\text { Systemic Constellation } \\
\text { Familienaufstellung }\end{array}$ & $* \mathrm{NR}$ & 5 & 5 & 2 \\
\hline $\begin{array}{l}\text { Google } \\
\text { Scholar }\end{array}$ & $\begin{array}{c}\text { Bert Hellinger } \\
\text { Family Constellation } \\
\text { Systemic Constellation } \\
\text { Familienaufstellung }\end{array}$ & $\begin{array}{c}2380 \\
2490 \\
130 \\
304\end{array}$ & 38 & - & 2 \\
\hline Total & & & 104 & 8 & 21 \\
\hline
\end{tabular}

Nota: Elaboración propia. *NR: Research Gate no muestra el número de registros encontrados en los resultados de la búsqueda.

Tras la búsqueda realizada se eliminaron artículos que no cumplían con los criterios de inclusión mediante la lectura crítica del título, resumen y documentos potencialmente relevantes. Del total de 112 referencias, se seleccionaron 21 documentos de interés. Para el análisis de los artículos seleccionados, se tomó como referencia la tabla diseñada por Bunge (2010) en su libro «Las Pseudociencias ¡vaya timo!» (Ver Tabla 2). 
Tabla 2 Comparación de las actitudes y actividades de los científicos y pseudocientíficos

\begin{tabular}{|c|c|c|c|c|c|c|}
\hline \multirow[b]{2}{*}{ Actitudes y actividades típicas } & \multicolumn{3}{|c|}{ Científico } & \multicolumn{3}{|c|}{ Pseudocientífico } \\
\hline & Si & No & $\mathbf{O P} *$ & Si & No & OP* \\
\hline $\begin{array}{l}\text { Admite su propia ignorancia y, por ende, la necesidad de mayor } \\
\text { investigación }\end{array}$ & $\mathrm{X}$ & & & & $\mathrm{X}$ & \\
\hline Considera que su propio campo es difícil y está lleno de lagunas & $\mathrm{X}$ & & & & $\mathrm{X}$ & \\
\hline Avanza mediante el planteamiento y resolución de nuevos problemas & $\mathrm{X}$ & & & & $\mathrm{X}$ & \\
\hline Recibe con agrado nuevas hipótesis y métodos & $\mathrm{X}$ & & & & $\mathrm{X}$ & \\
\hline Propone y ensayo nuevas hipótesis & $X$ & & & & & $\mathrm{X}$ \\
\hline Intenta descubrir o aplicar leyes & $\mathrm{X}$ & & & & $\mathrm{X}$ & \\
\hline Aprecia la unidad de la ciencia & $\mathrm{X}$ & & & & $\mathrm{X}$ & \\
\hline Se apoya en la lógica & $\mathrm{X}$ & & & & & $\mathrm{X}$ \\
\hline Utiliza la matemática & $\mathrm{X}$ & & & & & $\mathrm{X}$ \\
\hline Recoge o utiliza datos, especialmente cuantitativos & $\mathrm{X}$ & & & & & $\mathrm{X}$ \\
\hline Busca contraejemplos & $\mathrm{X}$ & & & & $\mathrm{X}$ & \\
\hline Inventa o aplica procedimientos objetivos de control & $\mathrm{X}$ & & & & $\mathrm{X}$ & \\
\hline Resuelve las disputas por medio del experimento o cálculo & $\mathrm{X}$ & & & & $\mathrm{X}$ & \\
\hline Recurre de manera sistemática a la autoridad & & $\mathrm{X}$ & & $\mathrm{X}$ & & \\
\hline Suprime o tergiversa datos no favorables & & $\mathrm{X}$ & & $\mathrm{X}$ & & \\
\hline Actualiza su información & $\mathrm{X}$ & & & & $\mathrm{X}$ & \\
\hline Busca comentarios críticos de otros & $\mathrm{X}$ & & & & $\mathrm{X}$ & \\
\hline Escribe artículos que pueden ser entendidos por cualquier persona & & $\mathrm{X}$ & & $\mathrm{X}$ & & \\
\hline Es probable que adquiera fama instantáneamente & & $\mathrm{X}$ & & $\mathrm{X}$ & & \\
\hline
\end{tabular}

*OP= Opcional. Fuente. Bunge, M. (2010). Las pseudoterapias jvaya timo!, Pamplona, España Laetoli S. A.

Para el propósito de esta investigación, la tabla fue modificada en ciertos criterios por los autores (Ver Tabla 3). Se eliminó: «intenta descubrir o aplicar leyes», «utiliza las matemáticas», «busca contraejemplos», y «escribe artículos que pueden ser entendidos por cualquier persona». Por el contrario, se añadió: «reconoce la existencia de otros modelos»y «se rige a organismos reguladores de control». Además, se modificó la redacción para facilitar la comprensión de constructos. Posteriormente, se analizó rigurosamente las CF mediante los criterios establecidos en la tabla modificada, con la información obtenida de los artículos seleccionados, este proceso se ilustra en la Tabla 3.

Tabla 3 Adaptación a la tabla de comparación de las actitudes y actividades de los científicos y pseudocientíficos

\begin{tabular}{|c|c|c|c|}
\hline & & \multicolumn{2}{|c|}{ Constelaciones familiares } \\
\hline \# & $\begin{array}{c}\text { Actitudes o } \\
\text { actividades típicas }\end{array}$ & SI & NO \\
\hline 1 & $\begin{array}{l}\text { Resalta la necesidad } \\
\text { de mayor } \\
\text { investigación }\end{array}$ & $\begin{array}{l}\text { (Mayer y Viviers, 2016) (Ramos y Ramos, } \\
\text { 2019) (Weinhold et al., 2013) (Alonso, } \\
\text { 2005) (Cohen 2008) }\end{array}$ & \\
\hline 2 & $\begin{array}{l}\text { Admite los vacíos } \\
\text { teóricos existentes en } \\
\text { el campo }\end{array}$ & $\begin{array}{l}\text { (Salters, 2013) (Hrnčić, 2017) (Weinhold } \\
\text { et al., 2013) (Mayer y Viviers, 2015) } \\
\text { (Mayer y Viviers, 2016) (Cohen 2008) }\end{array}$ & $\begin{array}{l}\text { (Geils y Edwards 2018) } \\
\text { (Alonso, 2005) }\end{array}$ \\
\hline 3 & $\begin{array}{l}\text { Avanza mediante el } \\
\text { planteamiento y } \\
\text { resolución de nuevos } \\
\text { problemas }\end{array}$ & $\begin{array}{l}\text { (Jaffenary et al., 2019) (Weinhold et al., } \\
\text { 2013) }\end{array}$ & (Geils y Edwards 2018) \\
\hline 4 & $\begin{array}{l}\text { Existe apertura hacia } \\
\text { nuevas hipótesis y } \\
\text { métodos }\end{array}$ & (Cohen 2008) & $\begin{array}{l}\text { (Stiefel et al., 2002) (McQuillin } \\
\text { y Welford, 2013) }\end{array}$ \\
\hline
\end{tabular}


Propone y ensaya nuevas hipótesis

6

Reconoce la unidad de la ciencia

$7 \quad$ Se apoya en la lógica

Recoge o utiliza datos cuantitativos
(Ramos y Ramos, 2019) (Weinhold et al., 2013) (McQuillin y Welford, 2013)

(Pritzker y Duncan, 2019) (Ramos y Ramos, 2019)

(Geils y Edwards 2018)

(Mayer y Viviers, 2016)

(Talarczyky, 2011) (Stiefel et al., 2002)

(Salters, 2013) (Cohen, 2006)

(Stiefel et al., 2002) (Cohen,

2008) (Duncan, 2017)

(McQuillin y Welford, 2013)

(Alonso, 2005)

(Pritzker y Duncan, 2019)

(Geils y Edwards 2018)

(Mayer y Viviers, 2016)

(Weinhold et al., 2013) (Hunger et al., 2014) (Hunger et al., 2014) (Jaffenary et al., 2019)
(Salters, 2013) (Talarczyky,

2011) (Stiefel et al., 2002)

(Cohen, 2008) (Duncan, 2017)

(McQuillin y Welford, 2013)

\section{Constelaciones familiares}

9 existencia de otros modelos

Inventa o aplica

10 procedimientos objetivos de control

Afronta las

controversias mediante métodos experimentales Depende de figuras

12 de autoridad para respaldar el modelo

Se rige a

13

organismos

reguladores de

control

Suprime o

14 tergiversa datos no favorables

Actualiza su

información

16

Busca comentarios

críticos de otros

Es probable que

17 adquiera reconocimiento social
(Pritzker y Duncan, 2019) (Mayer y

Viviers, 2016) (Salters, 2013) (Hunger et al., 2014) (Cohen, 2008) (McQuillin y Welford, 2013)

(Welford, 2019) (Weinhold et al., 2013) (Hunger et al., 2014); (Hunger et al., 2014)

(Weinhold et al., 2013) (Hunger et al., 2014) (Jaffenary et al., 2019) (Salters, 2013) (Stiefel et al., 2002) (Cohen, 2008)

(Alonso, 2005)

(Cohen, 2006) (Cohen, 2008)

(Mayer y Viviers, 2016)

(Hrnčić, 2017) (Talarczyky, 2011) (Cohen, 2008)

(Duncan, 2017)

(Geils y Edwards 2018) (Hrnčić, 2017)

(Alonso, 2005)

(Hunger et al., 2014)

(Welford, 2019) (Pritzker y Duncan, 2019)

(Hrnčić, 2017) (McQuillin y

Welford, 2013)

(Weinhold et al., 2013)

(Weinhold et al., 2013) (Welford, 2019) (Pritzker y Duncan, 2019).

(Geils y Edwards 2018) (Mayer y Viviers, 2016) (Alonso, 2005)

$* \mathrm{OP}=$ Opcional.

Fuente. Bunge, M. (2010). Las pseudoterapias jvaya timo!, Pamplona, España Laetoli S. A. 
Finalmente, con el objetivo de mantener unidad en la narrativa, los criterios de la tabla modificada fueron agrupados en los cinco criterios que deben cumplir los campos de conocimiento de la ciencia de la mente previamente descritos.

\section{RESULTADOS}

\subsection{CONCEPCIÓN GENERAL O FILOSOFÍA INHERENTE}

Salters (2013) argumenta que encontrar una fundamentación de la CF de Hellinger es difícil pues el autor era reacio a teorizar y enfatizaba su naturaleza fenomenológica. Tanto Hrnčić (2017) como Weinhold et al. (2013) plantean que carece de información sistematizada y de alcance, en referencia a sus efectos comparados con otras modalidades terapéuticas. Asimismo, se reconoce que los vacíos en la literatura son completados con nociones culturales y espirituales (Mayer y Viviers, 2015) (Mayer y Viviers, 2016). En contraste, para Geils y Edwards (2018) el fundamento teórico de las CF está en totalidad explicado desde las experiencias espirituales que elaboran de manera amplia y a profundidad estos fenómenos. Finalmente, Alonso (2005) es crítico al plantear que los campos de conocimiento con los cuales sustentan sus principios teóricos no tienen congruencia con la realidad, pero quienes la practican, actúan como si la hubiera.

También, Stiefel, Harris, Zollmann, (2002) expresan directamente que no está guiada por lógica o razonamiento, pues la técnica requiere una experiencia sensorial preconceptual, y considera al individuo conectado con planos espirituales superiores. La premisa principal de las CF se centra identificar lealtades inconscientes a nuestros antepasados, explicadas por dinámicas ocultas y conflictos transgeneracionales (Duncan, 2017). Dentro sus conceptos, existen nociones encontradas que corresponden a explicar el "enredo familiar" influido por un campo energético, siendo símil, al concepto de inconsciente colectivo (Salters, 2013). Alonso resalta la actitud de algunos de sus autores y practicantes, que promulgan su oficio tiñéndolo de esoterismo y atribuyéndole propiedades casi mágicas, principalmente cuando las explicaciones que brindan son de tipo místico, fenomenológico o cosmológico (2005).

La revisión mostró que la práctica de las CF no se rige a organismos reguladores, como propone Duncan (2017) en Estados Unidos no existe una asociación de profesionales que controle la práctica, entrenamiento o supervisión de los facilitadores de CF, asimismo, respecto a su supuesta filiación sistémica, las CF no son consideradas coherente con la teoría ni con la práctica de la terapia sistémica, por lo que entes reguladores de esta terapia no la respaldan (Talarczyky, 2011). Finalmente, Cohen pone en tela de juicio las modalidades de tratamiento sin regulación, que se promovieron como curas instantáneas a varias dolencias, reconociendo más tarde, mediante pruebas y estudios de caso, que su beneficio es limitado o incluso pueden llegar a ser perjudiciales (2008). 
Esta filosofía inherente puede conllevar a que las CF adquieran reconocimiento social debido a ciertas características. Mayer y Viviers (2016) manifiestan que las CF como forma de intervención ha ganado interés en contextos sudafricanos al igual que en Europa. Geils y Edwards (2018) expresan que los pacientes experimentan una evidente conexión de las CF con las vivencias espirituales, desde esa vivencia llaman la atención de sus lectores. Finalmente, Weinhold et al. (2013) proponen que, tras los resultados encontrados, proveen evidencia de efectividad, dándole una caracterización científica, desde la cual se le puede atribuir reconocimiento.

\subsection{METÓDICA O CONJUNTO DE MÉTODOS}

Sobre la metodología que emplean, dentro de los estudios cuantitativos revisados, 4 utilizan el método experimental para sustentar los efectos de las CF. Weinhold et al., en su estudio, que buscó demostrar eficacia a corto plazo, utilizando un grupo experimental y un grupo control, este ensayo controlado tuvo una muestra de 208 adultos y afirma demostrar la efectividad de las CF en una población no clínica (2013). Paralelamente y dentro del estudio de Weinhold et al., (Hunger, Bornhäuser, Link, Schweitzer y Weinhold, 2013) evalúan la eficacia de las CF sobre la experiencia dentro de sistemas sociales, los resultados muestran una mejor experiencia en los sistemas sociales de los individuos en comparación con el 73\% de un grupo en lista de espera. Conviene subrayar que, este proceso cuenta con limitaciones al no utilizar un grupo control apropiado. Además, explicitan que los cambios dentro del grupo control no pueden atribuirse a características específicas de la intervención.

Al año siguiente, Hunger, Weinhold, Bornhäuser, Link, y Schweitzer (2014) continúa con el estudio anterior, con el objetivo de examinar la estabilidad a medio y largo plazo de los efectos, para esto usa una muestra de 104 adultos, parte del grupo de estudio anterior, los resultados expresan eficacia de los procesos de CF a mediano y largo plazo en una población no clínica. En otro estudio, Jaffenary et al. (2019) Evaluaron la efectividad de las CF en la disminución de sintomatología en pacientes con dermatitis atópica y psoriasis, con 31 pacientes divididos en un grupo experimental y un grupo control, los resultados mostraron una disminución visible de sintomatología fisiológica.

De otro modo, 8 documentos utilizan una metodología cualitativa como la observación participante (Pritzker y Duncan, 2019), otros se centraban en fortalecer la conexión de los participantes con el alma familiar (Geils y Edwards, 2018). El estudio de Talarczyky (2011) señala que las herramientas científicas clásicas no pueden aplicarse al método de CF pues este es un espectáculo realizado sobre la base del psicodrama y de la hipnosis. Igualmente, Cohen (2008) sostiene que la constelación es un proceso conductor a una profundidad de la experiencia, abarcador de mente y espíritu. 
Esto indica como las CF no usan exclusivamente el método experimental, pero tienden a proponer y ensayar nuevas hipótesis en sus investigaciones, sobre esto, dentro de los procesos de valoración de eficacia se sometió a comprobación si los seminarios sobre CF mejoran el funcionamiento psicológico dándoles seguimientos longitudinales de 2 semanas y 4 meses (Weinhold et al., 2013) y desde una experiencia de estudio de caso se propone una nueva herramienta para medir constructos inherentes a la justificación teórica de las CF (Ramos y Ramos, 2019), Asimismo McQuillin y Welford (2013) defienden que las CF pueden brindar herramientas con las cuales curar a los sistemas familiares.

De la misma manera, se torna pertinente revisar si se aplican procedimientos objetivos de control en la investigación. Al respecto Weinhold et al., (2013) evalúan los cambios del proceso mediante el índice de cambio fiable (RCI) buscando consistencia interna de las medidas de resultados. Hunger et al., por su parte, hacen referencia a la utilización de un manual basado en sus propias observaciones y entrevistas con pioneros de las $\mathrm{CF}$, dicho manual explica cómo se debe realizar el proceso, donde monitoreaban y calificaban la ocurrencia de cada procedimiento estandarizado, sin embargo, no se pudo calcular dicha medida de una manera más fiable como a través del índice de Kappa $(2013,2014)$.

Cohen (2008) refiere, como limitación metodológica de su estudio, que la información recabada fue de cuestionarios archivados y recuerdos del investigador por lo que los datos no pudieron ser categorizados. Por el contrario, Mayer y Viviers (2016) explican que los facilitadores sudafricanos se refieren a las CF como un proceso basado en la fenomenología y creencia ancestral, lo que torna difícil la aplicación de un proceso de control. Salters (2013) menciona que la integración de las CF, el análisis transaccional (AT) y el juego de arena está guiada por un misterioso conocimiento interno que solo se revela dentro de esta práctica.

Frente a esto, Alonso se muestra crítico con las CF al referir que su sustento teórico necesita sistematización y filtrado, mediante pruebas empíricas, cuya falta de comprobación resulta casi bochornosa cuando se analiza el fenómeno de la supuesta transferencia de conocimiento a los representantes en la configuración (2005). Del mismo modo, Talarczyky (2011) sustenta que las CF al basarse en un contacto único con la persona que lo experimenta no cumplen con criterios básicos de un proceso psicoterapéutico.

\subsection{OBJETIVOS O METAS}

Una característica de un campo del conocimiento, es el planteamiento de mayor investigación, así Mayer y Viviers (2016) recomiendan mayor investigación del trabajo de CF en culturas diferentes a las asociadas a su origen y dentro de organizaciones, resaltando que no existe investigación empírica sobre sus principios. Por su parte Ramos y Ramos (2019) refieren que los resultados que avalan su efectividad, 
surgidos a partir de los estudios de caso deben ser contrastados con resultados a mayor escala. En contraste, Weinhold (2013), refiere que a pesar de demostrar la efectividad de los seminarios de CF esta necesita mayor evaluación, debido a que limitaciones en su método. En este sentido, Alonso (2005) plantea que los estudios deben presentar pruebas de sus postulados y evaluar la eficacia del procedimiento, haciendo hincapié en seguimientos a mediano y largo plazo, pues el efecto que genera, aparentemente, no es duradero.

Relacionado a lo anterior, con respecto a si actualiza su información, el aporte explicativo de Welford (2019) propone que las nuevas investigaciones sobre epigenética se pueden relacionar con las nociones transgeneracionales presentes en la CF. Así también en ciertas prácticas de consulta privada se ha combinado con otros métodos como el AT (Welford, 2019). Del mismo modo, Pritzker y Duncan (2019) manifiestan que la CF reúne varias teorías para discutir técnicas terapéuticas incompatibles con los discursos globalizadores hegemónicos actuales. En contraposición, Hrnčić (2017) expone que Hellinger sostenía que el facilitador no debe interesarse por la autonomía del cliente, ni documentar los efectos a largo plazo puesto que realizar un seguimiento significaba irrespetar la autonomía del paciente. Tampoco se alienta a los participantes de CF a seguir hablando de la constelación, sino a sostener y respetar lo sucedido en ese momento (McQuillin y Welford, 2013).

\subsection{FONDO ESPECÍFICO O SUPUESTOS RELACIONADOS A OTROS CAMPOS}

Pritzker y Duncan (2019) intentan, a partir de investigaciones experimentales, ubicarla dentro del campo de la psicoterapia como una teoría del Yo y de la familia. También se pretende definir a esta práctica como como una psicoterapia sistémica e integradora, e incluso resaltan la posibilidad de que pueda contribuir a enriquecer el cuerpo de las psicoterapias basadas en la evidencia (Ramos y Ramos, 2019).

Sobre la condición de reconocer la existencia de otros modelos, se encontró que los facilitadores de las CF se muestran abiertos a integrarla con otras intervenciones terapéuticas y prácticas espirituales para apoyar la curación (Mayer y Viviers, 2016). McQuillin y Welford (2013) reconocen que el trabajo de las CF se junta con la teoría y las técnicas de AT para tratar a sus clientes. De igual manera, en el estudio de Salters (2013) se menciona que en su práctica de AT ha explorado los juegos de arena y las constelaciones familiares.

En contraste, Stiefel et al. (2002) advierten que dado el trasfondo religioso de Hellinger, su terapia se ocupa de procesos que están más allá del espectro de la psicoterapia tradicional, abordando solamente procesos de la experiencia inmediata. también, Talarczyky (2011) llega a la conclusión de que la aplicación de CF no es psicoterapia pues carece de componentes básicos establecidos en la misma, además 
de resaltar los enunciados de la Asociación Sistémica en Alemania quienes promulgan que equiparar el trabajo de constelaciones con la terapia sistémica no sólo es incorrecto sino engañoso, y puede tornarse riesgoso tanto para la teoría sistémica como para el paciente, además, en cuanto al criterio de que si existe una búsqueda de comentarios críticos o la opinión de otros actores del campo, Cohen (2008) cuestiona la validez de la CF y otras terapias en problemas crónicos al plantearse como una forma efectiva de intervención con gran carga emocional de una sola sesión.

\subsection{PROBLEMÁTICA, O COLECCIÓN DE PROBLEMAS ABORDABLES}

Con base en el criterio de si al enfrentarse a datos no favorables los suprime o tergiversa, Geils y Edwards (2018) plantean que la dificultad encontrada en pacientes sintonizar con la experiencia se debe a falta de habilidad para percibir la experiencia de antepasados en sus sistemas culturales y espirituales, descartando así, que el fallo se centre en la técnica. Además, Hrnčić (2017) refiere que lo que determina profundamente los efectos de la terapia, su efectividad o perjuicio, es la competencia de los pacientes. Por su parte, Alonso, expresa que no se puede descartar totalmente ciertos constructos que fundamentan la naturaleza de las constelaciones, pero reconoce que no existe información suficiente para respaldarlos y, por ende, crítica que en su práctica actúen como si las hubieran (2005).

En cuanto al criterio de si existe apertura hacia nuevas hipótesis y métodos. Cohen (2008) propone que las construcciones reveladas en CF se podrían convalidar frente a recientes descubrimientos en la neurociencia cognitiva, y llegar a ofrecer un modelo teórico que pudiera resolver elementos aparentemente inexplicables. Oponiéndose a lo anterior McQuillin y Welford (2013) delimitan que el objetivo de toda constelación se centra en los principios de orden propuestos por Hellinger. Desde esta misma perspectiva fundamentada en aquel marco único, se propone la aplicación de CF para enfermedades médicas, trastornos psiquiátricos, discapacidad, infertilidad, victimización, perpetración de crímenes de guerra, adopción y problemas de pareja (Stiefel et al., 2002).

\section{DISCUSIÓN}

Desde la filosofía de la ciencia y concordando con Bunge (1985) se define un campo como pseudocientífico: 1) al estar basado en entes independientes de la materia como espíritus, Duncan (2017) propone la resolución de problemas identificando lealtades inconscientes a nuestros antepasados, sentando las bases de CF en aspectos intuitivos y pseudocientíficos; 2) compensar vacíos teóricos con conocimientos ancestrales, como Geils y Edwards (2018) al fundamentar la epistemología de la CF en la espiritualidad de la cultura Zulú y; 3) acoger problemáticas que no puedan ser abordadas mediante la 
investigación científica, en la literatura revisada se suponen dinámicas ocultas creadas conflictos transgeneracionales, creando pseudoproblemas insostenibles a comprobación.

Respecto a los objetivos, un campo del conocimiento que busque explicar el aspecto mental tiene que regirse a leyes objetivas de estudio. En CF hay ambigüedad puesto que existen estudios que intentan comprobar su efectividad (Weinhold et al., 2013), (Hunger et al., 2013), (Hunger et al., 2014), (Jaffenary et al., 2019), sin embargo, existe una notoria contraposición con otros (Salters, 2013), (Mayer y Viviers, 2015), (Alonso, 2005) (Stiefel et al., 2002), (Duncan 2017). Cohen refiere que el objetivo de las CF dista de la PBE puesto que, su función es la exploración de aspectos omitidos por las corrientes tradicionales (2008). En relación a lo metódico dichas especulaciones deben sobrevivir la comprobación bajo el método experimental para ser catalogadas como científicas, por tanto, bajo el parámetro de la filosofía de la ciencia, las CF entraría en el campo de lo pseudocientífico.

A parte del criterio de cientificidad, se debe establecer la disposición de la CF dentro del campo de la psicoterapia. Wampold (2019) refiere que la psicoterapia como práctica de curación está incrustada en un sistema de creencias, es decir, alojada en la teoría psicológica. Sin embargo, la literatura revisada indica que las CF se encuentran fundamentadas en las nociones espirituales y culturales vivenciadas por su autor Bert Hellinger por lo que desde este principio básico resulta inadecuada denominarla psicoterapéutica.

Es evidente que las CF utilizan terminología asociada a la de corriente sistémica. El modelo sistémico aborda la estructura y dinámica familiar desde las interacciones existentes de los miembros presentes y sus roles actuales, no la dinámica como un proceso espiritual cargado de causalidad mística, tampoco, postulan problemas que se encuentren en un plano temporal que resulta inaccesible ni relaciones causales con antepasados, como resultado, organismos reguladores sistémicos no lo respaldan (Talarczyky, 2011).

Abordar los accesos que intentan darle validez a las técnica CF resultó central para este proceso, esta validación significa una aproximación científica y sistemática de si un tratamiento funciona (APA Task Force on Evidence-Based Practice, 2006), desde ahí, sistematizar los resultados encontrados no es posible, pues la existencia de vacíos teóricos importantes en el modelo y falta de consenso entre los expertos en el área, impide la existencia de un objetivo común y, por ende, que sus resultados sean contrastables.

Individualmente, la validación implica: 1) uso de metodologías empíricas sofisticadas, los estudios reconocen sus limitaciones; 2) reconocimiento de variables extra terapéuticas, factores individuales en el paciente y las especificidades del ambiente, mismas que no reciben atención (APA Task Force on Evidence-Based Practice, 2006) (Weinhold et al., 2013), (Hunger et al., 2013), (Hunger et al., 2014), 
(Jaffenary et al., 2019). Ante lo anteriormente expuesto, ningún intento de validación debe considerarse concluyente cuando afirma algún nivel de efectividad.

Adicionalmente, el supuesto sustento fenomenológico del que habla la $\mathrm{CF}$, le hace renegar de la necesidad de validación (Cohen, 2008). No obstante, según Finlay (1999) un entendimiento enteramente fenomenológico implica una suspensión total de creencias previas centrándose en la experiencia inmediata y libre de arbitrariedades, criterios que no se cumplen a cabalidad en la ejecución de las CF. Si bien el fenómeno se analiza in situ, va direccionado por el criterio del facilitador, quien interpreta las expresiones de los participantes a través de preconcepciones que conforman las constelaciones perdiendo la característica descriptiva del método. Finalmente, la dificultad de liberarse de juicios, ser consciente de la propia intencionalidad y la tendencia a interpretar y así evitarla, este mecanismo no es enseñado ni aplicado dentro de las CF.

Cabe resaltar, que tras considerar el auge de estas prácticas en nuestro medio y los intentos por enmascararlas tras los modelos de tercera y cuarta generación, como se ha evidenciado en esta revisión, carecen de sustento teórico, validez y efectividad en su práctica. En consecuencia, resulta necesario llevar a cabo investigaciones que profundicen y den contexto a los postulados mencionados, logrando así, un mejor entendimiento de este fenómeno y abordar las limitaciones de esta investigación.

Finalmente, el desarrollo científico de cualquier modelo terapéutico implica tomar en cuenta la rigurosidad de sus métodos, sean protocolos básicos de intervención no medibles o bien intenten denominarse avances en teorías ya establecidas, enfatizando de forma particular los 3 componentes cruciales de la intervención, como: la frecuencia del tratamiento, la competencia del terapeuta e implementación de técnicas.

\section{AGRADECIMIENTOS}

A la Unidad de Posgrados y Laboratorio de Psicometría de la Universidad Católica de Cuenca.

\section{CONFLICTO DE INTERESES}

No existe ningún conflicto de Intereses entre los autores. 


\section{REFERENCIAS}

Alonso, Y. (2005). Las constelaciones familiares de Bert Hellinger: Un procedimiento psicoterapéutico en busca de identidad. International Journal of Psychology and Psychological Therapy, 5(1), 85-96. Recuperado de https://www.researchgate.net/publication/28116248

American Psychological Association, Presidential Task Force on Evidence-Based Practice. (2006). Evidence-based practice in psychology. American Psychologist, 61(4), 271-285. doi: 10.1037/0003066X.61.4.271

Bunge, M. (1985). Seudociencia e ideología. Madrid, España: Alianza Editorial.

Bunge, M. (2010). Las pseudociencias. ¡Vaya timo! Pamplona, España: Laetoli.

Cohen, D. (2006). "Family Constellations": An Innovative Systemic Phenomenological Group Process From Germany. The Family Journal, 14(3), 226-233. doi: 10.1177/1066480706287279

Cohen, D. (2008). Systemic family constellations and their use with prisoners serving long-term sentences for murder or rape (tesis doctoral). University of Saybrook, San Francisco, California. Recuperado de http://www.konsteliacijos-d.lt/multisites/konsteliacijos/images/stories/Simonos/cohen-2008dissertation-family-constellations.pdf

Duncan, W. (2017). Dinámicas Ocultas: Culture and Psy-Sociality in Mexican Family Constellations. Ethos, 45(4), 489-513. doi: 10.1111/etho.12175

Franke, U. (2017). The river never looks back: Historical and Practical Foundations of Bert Hellinger's Family Constellations (K. Leube, Trans.; 1st ed). Ebook edition. (Original work published 2003).

Finlay, L. (1999). Applying Phenomenology in Research: Problems, Principles and Practice. British Journal of Occupational Therapy, 62(7), 299-306. doi: 10.1177/030802269906200705

Fuchs, T., Messas, G., \& Stanghellini, G. (2019). More than Just Description: Phenomenology and Psychotherapy. Psychopathology, 52(1), 63-66. doi: 10.1159/000502266

Geils, C., \& Edwards, S. (2018). Extended family constellations workshop efficacy on intuition measure and experience. Journal of Psychology in Africa, 28(3), 224-228. doi: 10.1080/14330237.2018.1475527

Hellinger, B. (1999). Suffering is easier to bear than resolution. In Hellinger, B., \& Hovel, G. (Eds.), Acknowledging What Is (1st ed., pp 1-22). Zeig, Tucker y Co.

Hrnčić, J. (2017). Family constellation as a treatment for overcoming the consequences of violence on victims. Temida, 20(2), 219-240. doi: 10.2298/tem1702219h

Hunger, C., Bornhäuser, A., Link, L., Schweitzer, J., \& Weinhold, J. (2013). Improving experience in personal social systems through family constellation seminars: Results of a randomized controlled trial. Family Process, 53(2), 288-306. doi: 10.1111/famp.12051

Hunger, C., Weinhold, J., Bornhäuser, A., Link, L., \& Schweitzer, J. (2014). Mid- and Long-Term Effects of Family Constellation Seminars in a General Population Sample: 8- and 12-Month Follow-Up. Family Process, 54(2), 344-358. doi: org/10.1111/famp.12102 
Jafferany, M., Capec, S., Yaremkevych, R., Andrashko, Y., Capec, G., \& Petrek, M. (2019). Effects of family constellation seminars on itch in patients with atopic dermatitis and psoriasis: A patient preference controlled trial. Dermatologic Therapy, 32(6). doi.org/10.1111/dth.13100

Mayer, C. H., \& Viviers, A. (2016). Constellation work principles, resonance phenomena, and shamanism in South Africa. South African Journal of Psychology, 46(1), 130-145. doi: 10.1177/0081246315591339

Mayer, C. H., \& Viviers, R. (2015). Exploring Cultural Issues for Constellation Work in South Africa. Australian and New Zealand Journal of Family Therapy, 36(2), 289-306. doi: 10.1002/anzf.1103

McQuillin, J., \& Welford, E. (2013). How Many People Are Gathered Here? Group Work and Family Constellation Theory. Transactional Analysis Journal, 43(4), 352-365. doi: 10.1177/0362153713519743

Pérez-Álvarez, M. (2019). La Psicoterapia Como Ciencia Humana, Más Que Tecnológica. Papeles del Psicólogo, 40(1), 1-14. doi: 10.23923/pap.psicol2019.2877

Pritzker, S., \& Duncan, W. (2019). Technologies of the Social: Family Constellation Therapy and the Remodeling of Relational Selfhood in China and Mexico. Culture, Medicine and Psychiatry, 43(3), 468495. doi: 10.1007/s11013-019-09632-x

Ramos, S., \& Ramos, J. (2019). Process of Change and Effectiveness of Family Constellations: A Mixed Methods Single Case Study on Depression. The Family Journal, 27(4), 418-428. doi: $10.1177 / 1066480719868706$

Salters, D. (2013). Sandplay and Family Constellation. Transactional Analysis Journal, 43(3), 224-239. https://doi.org/10.1177/0362153713509955

Stiefel, I., Harris, P., \& Zollmann, A. (2002). Family Constellation - A Therapy Beyond Words. Australian and New Zealand Journal of Family Therapy, 23(1), 38-44. doi: 10.1002/j.1467-8438.2002.tb00484.x

Stones, B. (2006). A Brief History of Bert Hellinger's Family Constellations. Self \& Society, 33(4), 5-9. doi: 10.1080/03060497.2006.11086254

Talarczyk, M. (2011). Family constellation method of Bert Hellinger in the context of the code of ethics for psychotherapists. Archives of Psychiatry and Psychoterapy, 3(1), 65-74. Recuperado de http://www.archivespp.pl/uploads/images/2011_13_3/65Talarczyk_APP3_2011.pdf

Wampold, B. E. (2019). Introduction. In B. E. Wampold, Theories of Psychotherapy Series. The basics of psychotherapy: An introduction to theory and practice (p. 3-15). American Psychological Association. doi: $10.1037 / 0000117-001$

Welford, E. (2019). Healing the Fallout From Transgenerational Trauma: Supporting Clients in Making Peace With Their History. Transactional Analysis Journal, 49(4), 324-338. doi: 10.1080/03621537.2019.1650233

Weinhold, J., Hunger, C., Bornhäuser, A., Link, L., Rochon, J., Wild, B., \& Schweitzer, J. (2013). Family constellation seminars improve psychological functioning in a general population sample: Results of a randomized controlled trial. Journal of Counseling Psychology, 60(4), 601-609. doi: 10.1037/a0033539 
Wojnar, D., \& Swanson, K. (2007). Phenomenology: An Exploration. Journal of Holistic Nursing, 25(3), 172-180. doi: 10.1177/0898010106295172 